\title{
Group Testing with Random Pools: optimal two-stage algorithms
}

\author{
Marc Mézard, Cristina Toninelli
}

\begin{abstract}
We study Probabilistic Group Testing of a set of $N$ items each of which is defective with probability $p$. We focus on the double limit of small defect probability, $p \ll 1$, and large number of variables, $N \gg 1$, taking either $p \rightarrow 0$ after $N \rightarrow \infty$ or $p=1 / N^{\beta}$ with $\beta \in(0,1 / 2)$. In both settings the optimal number of tests which are required to identify with certainty the defectives via a two-stage procedure, $\bar{T}(N, p)$, is known to scale as $N p|\log p|$. Here we determine the sharp asymptotic value of $\bar{T}(N, p) /(N p|\log p|)$ and construct a class of two-stage algorithms over which this optimal value is attained. This is done by choosing a proper bipartite regular graph (of tests and variable nodes) for the first stage of the detection. Furthermore we prove that this optimal value is also attained on average over a random bipartite graph where all variables have the same degree, while the tests have Poisson-distributed degrees. Finally, we improve the existing upper and lower bound for the optimal number of tests in the case $p=1 / N^{\beta}$ with $\beta \in[1 / 2,1$ ).
\end{abstract}

Index Terms-Group testing, reconstruction algorithms

\section{INTRODUCTION}

The aim of Group Testing is to detect an unknown subset of defective (also referred to as positive or active) items out of a set of objects by means of queries (the tests) in the most efficient way. In other words we are given a set of objects, $\mathcal{O}$, which contains an unknown subset of defectives, $\mathcal{D}$, and the task is to identify $\mathcal{D}$ by means of the fewest possible number of tests. Tests are queries of the form "Does the pool $\mathcal{Q}$ (where $\mathcal{Q}$ is a subset of $\mathcal{O}$ ) contain at least one positive item?". This problem was originally introduced in relation with efficient mass blood testing [1]. Afterwards, it has been also applied in a variety of situations in molecular biology: blood screening for HIV tests [2], screening of clone libraries [3], [4], sequencing by hybridization [5], [6], ... Furthermore it has proved relevant for fields other than biology including quality control in

- M.Mézard is with CNRS, LPTMS, Batiment 100, Université de Paris Sud 91405 Orsay - France (e-mail: mezard@lptms.upsud.fr);

- C.Toninelli is with CNRS, LPMA, Boite courrier 188, 4 Pl. Jussieu, Univ.Paris VI-VII, France (e-mail: ctoninel@ccr.jussieu.fr)

This work has been supported in part by the EC grant "Evergrow", IP 1935 of FET-IST. product testing [7], searching files in storage systems [8], data compression [9] and more recently in the context of data gathering in sensor networks [10]. We refer to [11], [12] for reviews on the different applications of GT. Here we will deal with the very much studied goldstandard case, namely the idealized situation in which tests are perfect: there can be neither false positives nor false negatives in the test answers. It is important to keep in mind for future work that, however, in many biological applications one should include the possibility of errors in the test answers.

Before presenting our results we recall some standard classifications of GT problems. First of all a GT problem can be either Combinatorial or Probabilistic. Combinatorial GT refers to the situation in which $\mathcal{D}$ can be any member of a predetermined class of sets in $\mathcal{O}$. The task is here to find the algorithm which requires the minimal number of tests to determine $\mathcal{D}$ in the worst case. In probabilistic GT we are given a configuration space $S$ and a probability distribution $\mu$ on $S$ and the set of objects $\mathcal{O}$ (and therefore the corresponding $\mathcal{D}$ ) is chosen in $S$ according to $\mu$. In this case the task is to optimize the expected (with respect to $\mu$ ) number of tests required to determine $\mathcal{D}$. Furthermore in 
both combinatorial and probabilistic GT there is an additional classification which concerns the number of stages, i.e. parallel queries, in the detection procedure. For one-stage (or fully non-adaptive) algorithms all tests are specified in advance: the choice of the pools $\{\mathcal{Q}\}$ does not depend on the outcome of the tests (and therefore does not depend on $\mathcal{O}$ ). For several biological applications a non-adaptive procedure would in principle be the best one. Indeed the test procedure can be destructive for the objects and repeated tests on the same sample require more sophisticated techniques. However the number of tests required by fully non-adaptive algorithms can be much larger than for adaptive ones. The best compromise for most screening procedures [13] is therefore to consider two-stage algorithms with a first stage containing a set of predetermined pools (tested in parallel) and a second stage whose pools are chosen depending on the outcomes of the first stage (and therefore on the choice of $\mathcal{O})$. For Probabilistic GT the only possibility to detect all defectives with such a procedure is to choose a trivial two-stage algorithms [13] which individually tests on the second stage all the variables which are left undetermined by the first stage. Here we will consider Probabilistic Group Testing when $\mu$ is Bernoulli product measure and we will analyze the performance of two stage algorithms as a function of the overall number of objects, $N$, and of the probability that a chosen object is defective, $p$. In particular we will analyze the relevant limit of small $p$ and large $N$, which has already been investigated in ( [13]-[16]). A detailed account of our new contributions follows.

\section{Notation AND RESUlts}

We consider Probabilistic Group Testing in the Bernoulli p-scheme: the configuration space is $S=\{0,1\}^{N}$, namely the set of all vectors $X=\left(x_{1}, \ldots, x_{N}\right)$ with $x_{i}=\{0,1\}$, and the probability measure is Bernoulli product measure $\mu_{p}$ with marginal $\mu_{p}\left(x_{i}=1\right)=p$, namely $\mu_{p}(X)=\prod_{i=1}^{N} p^{x_{i}}(1-p)^{1-x_{i}}$. For a given choice of $X$ we say that variable $i$ is (is not) defective or positive if $x_{i}=1\left(x_{i}=0\right)$.
A test of the type "Does pool $\mathcal{Q}$ contain at least a defective?" corresponds here to asking whether the value of the random variable constructed as an OR function among the variables of the pool equals one or zero. More precisely we will call "pool $a$ " an $N$ component binary vector $P_{a}=\left(c_{1 a}, c_{2 a}, \ldots, c_{N a}\right)$ with $c_{i, a} \in\{0,1\}$ and we will say that variable $i$ belongs (does not belong) to pool $a$ if $c_{i, a}=1\left(c_{i, a}=0\right)$. With this notation we will call "test $a$ " the random variable $T_{a} \in\{0,1\}$ with $T_{a}=0$ if $c_{i, a} x_{i}=0$ for all $i=(1, \ldots, N), T_{a}=1$ otherwise. In other words $T_{a}$ is the OR function among the variables that belong to pool $a$.

For a given choice of the variables, $X$, and a set of $M$ pools, $\left\{P_{a}\right\}, a=1, \ldots M$, we say that: (a) variable $i$ is a sure zero if there exists at least one $a \in(1, \ldots, M)$ such that: $i$ belongs to pool $a$ and $T_{a}=0(\mathrm{~b})$ variable $i$ is a sure one if there exists at least one $a \in(1, \ldots, M)$ such that: $i$ belongs to pool $a, T_{a}=1$ and all the other variables $j, j \neq i$, which belong to pool $a$ are sure zeros.

It is obvious that if $i$ is a sure zero then $x_{i}=0$ and if $i$ is a sure one then $x_{i}=1$. Note that however the converse is not true: there can be a zero (one) variable that is not a sure zero (sure one, respectively). Indeed, any given choice of $X, M$ and $\left\{P_{a}\right\} a=(1, \ldots, M)$, identifies the following subsets of $\mathcal{V}:=\{1, \ldots, N\}$.

(i) The zeros and the ones

$$
\begin{gathered}
\mathcal{Z}:=\left(i: i \in \mathcal{V} ; x_{i}=0\right) \\
\mathcal{D}:=\left(i: i \in \mathcal{V} ; x_{i}=1\right)=\mathcal{V} \backslash \mathcal{Z} ;
\end{gathered}
$$

(i) The sure zeros and the sure ones

$$
\mathcal{Z} \supset \mathcal{S}_{0}:=\left(i: i \in \mathcal{V} ; \quad \prod_{a=1}^{M}\left(T_{a}\right)^{c_{i, a}}=0\right)
$$

$\mathcal{D} \supset \mathcal{S}_{1}:=\left(i: i \in \mathcal{V} ; \sum_{a=1}^{M} c_{i, a} x_{i} \prod_{j \neq i}\left(\mathbb{I}_{\mathcal{S}_{0}}(j)\right)^{c_{j, a}}>0\right)$

(here and throughout this paper we define $0^{0}=1$ and $\mathbb{I}_{A}$ stands for the characteristic function of set $A$ ); .

(iii) The undetermined zeros and the undetermined ones

$$
\mathcal{U}_{0}:=\mathcal{Z} \backslash \mathcal{S}_{0}, \quad \mathcal{U}_{1}:=\mathcal{D} \backslash \mathcal{S}_{1}
$$


The two-stage algorithms that we consider are composed by a first stage of parallel tests and a second stage of individual tests over the variables whose value has been left undetermined by the first stage. Therefore the choice of the algorithm is completely defined by fixing the number of tests in the first stage, $M \in \mathbb{N}$, and by choosing the pools $\left\{P_{a}\right\}, a=1, \ldots, M$. The latter corresponds to fixing an $N \times M$ matrix $C_{N, M}$ with binary entries $c_{i, a} \in\{0,1\}$ which give the i-th component of vector $P_{a}$. This will be called the connectivity matrix. In other words, the choice of the algorithm corresponds to fixing a couple $\left(M, C_{N, M}\right)$, namely choosing a bipartite graph $G=\mathcal{G}\left(C_{N, M}\right)$ with $N$ variable nodes and $M$ test nodes. The number of tests required to identify the defectives (i.e. to decode the value of $X), T\left(X, M, C_{N, M}\right)$, is therefore given by the number of tests in the first stage plus the number of variables which are left undetermined by them, namely

$$
T\left(X, M, C_{N M}\right):=M+\left|\mathcal{U}_{0}\right|+\left|\mathcal{U}_{1}\right| .
$$

Note that $\mathcal{U}_{0}$ and $\mathcal{U}_{1}$ depend in general on $X, M$ and $C_{N, M}$. We will denote by $T_{M, C_{N, M}, p}$ the mean of $T\left(X, M, C_{N, M}\right)$ over the Bernoulli distribution $\mu_{p}$ for $X$, namely

$$
T_{M, C_{N, M}, p}:=M+\sum_{X \in S} \mu_{p}(X)\left(\left|\mathcal{U}_{0}\right|+\left|\mathcal{U}_{1}\right|\right) .
$$

In this probabilistic setting the first important issue is to determine the optimal value $\bar{T}(N, p)$ of $T_{M, C_{N, M}, p}$ over all two-stage algorithms, i.e. over all choices of $M$ and $C_{N, M}$

$$
\bar{T}(N, p):=\min _{M, C_{N, M}} T_{M, C_{N, M}, p}
$$

where minimization is restricted to $M=$ $(1, \ldots, N)$ (it is obvious that the optimal value can never be attained at $M \geq N+1$ ).

Here we will study this problem in the relevant limit of small defective probability, $p \ll 1$, which has already been investigated in [13]-[16]. We will denote by $\lim _{N \rightarrow \infty \mid \beta}$ the limit where $N$ goes to $\infty, p$ goes to zero, with $p=N^{-\beta}$ and $\beta>0$, i.e.

$$
\lim _{N \rightarrow \infty \mid \beta} f(N, p):=\lim _{N \rightarrow \infty} f\left(N, N^{-\beta}\right) .
$$

We will also study the $\operatorname{limit} \lim _{p \rightarrow 0} \lim _{N \rightarrow \infty}$ and, in order to lighten the presentation of our results, we will refer to this case as the $\beta=0$ case:

$$
\lim _{N \rightarrow \infty \mid 0} f(N, p):=\lim _{p \rightarrow 0} \lim _{N \rightarrow \infty} f(N, p) .
$$

Our main contributions are the following results for the asymptotics of $\bar{T}(N, p)$, which will be proved in Section 4 and 5 , respectively.

Theorem 1: When $\beta \in[0,1 / 2)$,

$$
\lim _{N \rightarrow \infty \mid \beta} \frac{\bar{T}(N, p)}{N p|\log p|}=\frac{1}{(\log 2)^{2}} .
$$

Theorem 2: When $\beta \geq 1 / 2$,

$$
\frac{1}{(\log 2)^{2}} \leq \lim _{N \rightarrow \infty \mid \beta} \frac{\bar{T}(N, p)}{N p|\log p|} \leq e .
$$

To our knowledge the best previously known bound for $0<\beta<1$ were

$$
\frac{1}{\log 2} \leq \lim _{N \rightarrow \infty \mid \beta} \frac{\bar{T}(N, p)}{N p|\log p|} \leq \frac{4}{\beta}
$$

which have been obtained in [14]: the lower bound via the information theoretic bound and the upper bound by the explicit construction of a decoding algorithm based on a random choice of the pools.

Our results determine the sharp asymptotics of $\bar{T}(N, p) /(N p|\log p|)$ for the cases $p=N^{-\beta}$ with $\beta \in(0,1 / 2)$ and for the cases $p \rightarrow 0$ after $N \rightarrow \infty$. Furthermore, they sharpen the previously existing bounds for $p=N^{-\beta}$ with $1 / 2 \leq \beta<1$.

A second relevant issue is the explicit construction of an asymptotically optimal algorithm, namely the identification of a family of couples $\left(M, C_{N, M}\right)$ such that

$$
\lim _{N \rightarrow \infty \mid \beta} \frac{T_{M, C_{N, M}, p}}{N p|\log p|}=\lim _{N \rightarrow \infty \mid \beta} \frac{\bar{T}(N, p)}{N p|\log p|} .
$$

Here, for each $\beta$ with $0 \leq \beta<1 / 2$, we identify a ( $\beta$-dependent) family of couples $\left(M, C_{N, M}\right)$ which satisfies (9). Let

$$
\bar{L}=:[|\log p| / \log 2]
$$




$$
\bar{M}=:\left[N p|\log p| /(\log 2)^{2}\right]
$$

where $[x]$ stands for the integer part of $x$. We construct a pooling design based on a "regularregular " bipartite graph with $N$ variable nodes, $\bar{M}$ test nodes, $\bar{L}$ tests per variable and $\bar{K}=$ $N \bar{L} / \bar{M}$ variables per tests, and with girth (i.e. length of the shortest graph cycle) at least 6 . This means that the corresponding connectivity matrix satisfies the following constraints

$$
\begin{gathered}
\forall a \in(1, \ldots, M): \sum_{j=1}^{N} c_{j, a}=\bar{K} \\
\forall i \in(1, \ldots, N): \sum_{b=1}^{M} c_{i, b}=\bar{L} \\
\sum_{1 \leq j<l \leq N} \sum_{1 \leq d<b \leq M} c_{j, b} c_{j, d} c_{l, b} c_{l, d}=0
\end{gathered}
$$

In Section 4 we will prove that any such connectivity matrix is asymptotically optimal, namely

Theorem 3: Let $C_{N, \bar{M}}^{\bar{L}}$ be such that conditions (12), (13) and (14) are satisfied. If $0 \leq \beta<1 / 2$, then:

$$
\lim _{N \rightarrow \infty \mid \beta} \frac{T_{\bar{M}, C_{N, \bar{M}}^{\bar{L}}, p}}{N p|\log p|}=\frac{1}{(\log 2)^{2}} .
$$

Notice that the family of graphs satisfying the requested properties is non-empty under the conditions for $\beta$ stated in the theorem, thanks to a constructive procedure found in [17], as we shall discuss in the proof.

Furthermore we have proved that the optimal value is also attained asymptotically by some random pool designs whose construction is much simpler than the one of [17]. Let $P_{N, M, L}^{\mathcal{R}-\mathcal{P}}$ denote the distribution of bipartite "regular-Poisson" graphs with $N$ variable nodes, $M$ test nodes, and a fixed number of tests, $L$, randomly connected to each variable node. Explicitly:

$$
P_{N, M, L}^{\mathcal{R}-\mathcal{P}}\left(C_{N, M}\right):=\prod_{i=1}^{N} P\left(c_{i, 1}, \ldots, c_{i, M}\right)
$$

with

$$
P\left(c_{i, 1}, \ldots, c_{i, M}\right):= \begin{cases}\frac{1}{\left(\begin{array}{c}
M \\
L
\end{array}\right)} & \text { if } \sum_{a=1}^{M} c_{i, a}=L \\
0 & \text { otherwise. }\end{cases}
$$

Note that, when one takes the large $N$ limit with $L \ll N$ and $L \ll M$, the degrees of the tests become iid random variables with a Poisson distribution of mean $K=N L / M$. If we make the choice $L=\bar{L}$ and $M=\bar{M}$ as in (10) and (11) the following result, whose proof is provided in section [5, holds

Theorem 4: When $0 \leq \beta<1 / 2$

$$
\lim _{N \rightarrow \infty \mid \beta} \sum_{C_{N, \bar{M}}} P_{N, \bar{M}, \bar{L}}^{\mathcal{R}-\mathcal{P}}\left(C_{N, \bar{M}}\right) \frac{T_{\bar{M}, C_{N, \bar{M}}, p}}{N p|\log p|}=\frac{1}{(\log 2)^{2}} .
$$

Finally, we provide a random class of connectivity matrices for which our upper bound in (7) for the case $p=1 / N^{\beta}$ with $1 / 2 \leq \beta<1$ is attained. Let $P_{N, M, L}^{\mathcal{P}-\mathcal{P}}$ denote the distribution of random bipartite graphs with $N$ variable nodes, $M$ test nodes and $l$ tests per variable $(k$ variables per test), with $l$ Poisson distributed and with mean $L$ ( $k$ Poisson distributed with mean $K=N L / M)$, namely

$$
P_{N, M, L}^{\mathcal{P}-\mathcal{P}}\left(C_{N, M}\right)=\prod_{a=1}^{M} \prod_{i=1}^{N}\left(\frac{L}{M}\right)^{c_{i, a}}\left(1-\frac{L}{M}\right)^{1-c_{i, a}} .
$$

If we make the choice $L=\widetilde{L}$ and $M=\widetilde{M}$ with

$$
\begin{gathered}
\widetilde{L}=:[e|\log p|] \\
\widetilde{M}=:[e N p|\log p|]
\end{gathered}
$$

the following result, whose proof is given in section 6, holds

Theorem 5: When $0 \leq \beta<1$

$$
\lim _{N \rightarrow \infty \mid \beta} \sum_{C_{N, \widetilde{M}}} P_{N, \widetilde{M}, \widetilde{L}}^{\mathcal{P}-\mathcal{P}}\left(C_{N, \widetilde{M}}\right) \frac{T_{\widetilde{M}, C_{N, \widetilde{M}}, p}}{N p|\log p|}=e .
$$

Furthermore, the choice (20)-(21) for the couple $(M, L)$ is optimal over all the PoissonPoisson distributions, namely

Remark 1: When $0 \leq \beta<1$, for any $M, L$

$$
\lim _{N \rightarrow \infty \mid \beta} \sum_{C_{N, \bar{M}}} P_{N, M, L}^{\mathcal{P}-\mathcal{P}}\left(C_{N, M}\right) \frac{T_{M, C_{N, M}, p}}{N p|\log p|} \geq e .
$$

Note that the Poisson-Poisson distribution had already been used in [14] to obtain the upper bound on $\bar{T}(N, p)$ which we have recalled in formula (8). Here, by optimizing the 
choice of the parameters $L$ and $M$ for $P_{N, M, L}^{\mathcal{P}-\mathcal{P}}$ we ameliorate the upper bound (8) which was obtained in [14] by choosing $M=4 N p|\log (p)|$ and $L=2|\log p|$.

Furthermore the result of Remark 1 together with Theorem 1 imply that the optimal value for $\beta \in[0,1 / 2)$ can never be attained on the class of Poisson-Poisson distributions, while it can be attained both on the class of regular-Poisson distributions and on the class of regular-regular graphs with girth at least 6 (see Theorem 4).

The outline of the paper is the following. In section 3 we establish a lower bound on $T_{M, C_{N, M}, p}$ (Theorem 6) which holds for any $N$ and $p \rightarrow 0$. In section 4 we prove Theorem 3 which, together with Theorem 6, completes the proof of Theorem 1 and identifies a set of algorithms for which the asymptotic value of $T_{M, C_{N, M}, p}$ is attained if $\beta \in[0,1 / 2)$. In section 5 we prove Theorem 4 which identifies a different class of random algorithms over which this asymptotic value is also attained. In Section 6 we prove Theorem 5 which, together with Theorem 6, completes the proof of Theorem 2 and identifies an algorithm over which our upper bound on $T_{M, C_{N, M}, p}$ is attained when $1 / 2 \leq \beta<1$.

\section{LOWER BOUND ON $\bar{T}(N, p)$}

In this section we prove the following lower bound on $\bar{T}$ which holds for any $N$ whenever we let $p \rightarrow 0$.

Theorem 6:

$$
\lim _{p \rightarrow 0} \frac{\bar{T}(N, p)}{N p|\log p|} \geq \frac{1}{(\log 2)^{2}} .
$$

When one takes the limit $\lim _{N \rightarrow \infty \mid \beta}$ with $\beta \in$ $[0,1)$, Theorem 6 improves the previously existing lower bounds [14] on $\bar{T}(N, p)$. Furthermore for all the cases $\beta \in[0,1 / 2)$ it allows, together with Theorem 4 to determine the exact value of $\lim _{N \rightarrow \infty \mid \beta} \bar{T} /(N p|\log p|)$. On the other hand when $\beta \geq 1$ better bounds then the one given by our (24) already exist [13], [14].

Let $q:=(1-p)$ and $Z_{N}^{+}:=\{0,1, \ldots, N\}$, we define $B_{M, C_{N, M}, p}$ and the function $A_{p}:\left(Z_{N}^{+}\right)^{N} \rightarrow$
$\mathbb{R}$ as

$$
\begin{aligned}
& B_{M, C_{N, M}, p}:=q \sum_{i=1}^{N} \prod_{a=1}^{M}\left(1-q^{d_{a}-1}\right)^{c_{i, a}} \\
& A_{p}(\vec{m}):=\sum_{i=1}^{N} \frac{m_{i}}{i}+q 0^{m_{1}} \exp \left(-\sum_{i=2}^{N} m_{i} a_{p}^{i}\right)
\end{aligned}
$$

where $d_{a}$ is the degree of test $a$, i.e.

$$
d_{a}:=\sum_{l=1}^{N} c_{l, a}
$$

and, for $i=2, \ldots N$,

$$
a_{p}^{i}:=\left|\log \left(1-(1-p)^{i-1}\right)\right| .
$$

We also let

$$
\begin{aligned}
\bar{A}_{p} & :=\min _{\vec{m} \in\left(Z_{N}^{+}\right)^{N}} A_{p}(\vec{m}) \\
U(p) & :=\min _{r \in[2, \infty)} \frac{1}{r\left|\log \left(1-(1-p)^{r-1}\right)\right|} \\
c(p) & :=\min _{w \in[0, \infty)}\left(U(p) w+(1-p) e^{-w}\right)
\end{aligned}
$$

In order to prove Theorem 6 we will use the following results, whose proofs are postponed to the next sections.

\section{Lemma 1:}

(a) For any choice of $M$ and $C_{N, M}$ the expected number of undetected zeros, $\mathcal{U}_{0}$, is lower bounded by

$$
\sum_{X} \mu_{p}(X)\left|\mathcal{U}_{0}\right| \geq B_{M, C_{N, M}, p}
$$

(b) If the girth of the graph $\mathcal{G}\left(C_{N, M}\right)$ is larger or equal to 6 , (31) holds as an equality.

Lemma 2:

$$
\min _{M, C_{N, M}}\left(M+B_{M, C_{N, M}, p}\right) \geq N \bar{A}_{p} .
$$

Lemma 3:

$$
\bar{A}_{p} \geq \min (1, c(p)) .
$$

Claim 1: When $p \rightarrow 0, U(p)=\frac{p}{(\log 2)^{p}}+\Theta\left(p^{2}\right)$.

Proof of Theorem 6 . 
By using definition (2), Lemma 1 and the where trivial inequality $\sum_{X} \mu_{p}(X)\left|\mathcal{U}_{1}\right| \geq 0$, we get

$$
T_{M, C_{N, M}, p} \geq M+B_{M, C_{N, M}, p} .
$$

Notice that for all the choices of $p$ that we consider, the bound will not suffer from the fact that we neglected the contribution from undetermined ones. This can be seen from the facts

$$
\sum_{X} \mu_{p}(X)\left|\mathcal{U}_{1}\right| \leq \sum_{X} \mu_{p}(X)|\mathcal{D}|=p N
$$

and $T_{M, C_{N, M}, p} \geq-N p \log _{2} p$ (this is the information theoretic lower bound [14]), which imply that for any $\beta \in[0,1)$ :

$$
\lim _{N \rightarrow \infty \mid \beta} \frac{\sum_{X} \mu_{p}(X)\left|\mathcal{U}_{1}\right|}{T_{M, C_{N, M}, p}}=0 .
$$

Since (33) holds for any $\left(M, C_{N, M}\right)$, by using Lemma 2 and 3 it follows immediately that

$$
\bar{T}(N, p) \geq N \min (1, c(p)) .
$$

From definition (30), as an immediate corollary of Claim 1, we get

$c(p)=\frac{p|\log p|}{(\log 2)^{2}}+(1-2|\log (\log 2)|) \frac{p}{(\log 2)^{2}}+o(p)$

in the limit $p \rightarrow 0$. By gathering the results (34) and (35) the proof of Lemma (3) is concluded. Furthermore, we get the following lower bound for the corrections

$$
\frac{1-2|\log (\log 2)|}{(\log 2)^{2}} \leq \frac{\bar{T}(N, p)-N p|\log p|(\log 2)^{-2}}{N p}
$$

\subsection{Proof of Lemma 1}

(a) By definition the set of undetected zeros, $\mathcal{U}_{0}$, contains all the variables $i$ such that $x_{i}=0$ and $T_{a}=1$ for any $a$ such that $c_{i, a}=1$, i.e. $i$ belongs only to pools containing at least a variable equal to one. Therefore:

$$
\sum_{X} \mu_{p}(X)\left|\mathcal{U}_{0}\right|=\sum_{i=1}^{N} \sum_{X} \mu_{p}(X)\left(1-x_{i}\right) \prod_{a=1}^{M} W_{i, a}(X)
$$

$$
W_{i, a}(X):=\left(1-\prod_{\substack{j=1, \ldots, N \\ j \neq i}}\left(1-x_{j}\right)^{c_{j, a}}\right)^{c_{i, a}} .
$$

Since $W_{i, a}(X)$ does not depend on $x_{i}$ we can immediately perform the average over this variable for each term of the sum in (37). Then, for each given $i$, we introduce the partial order $\prec_{i}$ according to which $x \prec_{i} x^{\prime}$ if and only if $x_{j} \leq x_{j}^{\prime}$ for all $j \in\{(1, \ldots, N) \backslash i\}$. For any $C_{N, M}$ and for any $a \in(1, \ldots, M), W_{i, a}$ is a nondecreasing function with respect to this partial order, namely $x \prec_{i} x^{\prime}$ implies that $W_{i, a}(X) \leq$ $W_{i, a}\left(X^{\prime}\right)$. Therefore inequality (31) follows by applying FKG inequality [18] to each term of the sum in (37). In other words, we have simply used the positive correlation among the events that there exists at least one variable equal to one in two (or more) intersecting pools.

(b) If the bipartite graph has girth at least 6 , i.e. if $M, C_{N, M}$ are such that for any couple of variables there exists at most one test which contains both of them (see condition (14)), the events defined above are independent. Therefore (31) holds with the equality sign.

\subsection{Proof of Lemma 2}

Given a choice $\left(M, C_{N, M}\right)$, we define for each variable $i$ the vector $\vec{m}^{i}=\left(m_{1}^{i}, \ldots, m_{N}^{i}\right) \in$ $\left(Z_{N}^{+}\right)^{N}$ where $m_{j}^{i}$ denotes the number of tests which contain variable $i$ and globally contain $j$ variables

$$
m_{j}^{i}:=\sum_{a=1}^{M} c_{i, a} \delta_{j, d_{a}}
$$

where $d_{a}$ is defined in (27). (and $\delta$ is the Kronecker $\delta$ ). Then we define for each $\vec{m}=$ $\left(m_{1}, \ldots, m_{N}\right) \in\left(Z_{N}^{+}\right)^{N}$ a density, $f(\vec{m})$, such that $N f(\vec{m})$ is the number of variables $i$ for which $\vec{m}^{i}=\vec{m}$. With this notation we can rewrite the number of tests, $M$, and the definition (25) for $B_{M, C_{N, M}, p}$ as

$$
\begin{aligned}
& M=N \sum_{\vec{m}} f(\vec{m}) \sum_{j=1}^{N} \frac{m_{j}}{j} \\
& B_{M, C_{N, M}, p}=N q \sum_{\vec{m}} f(\vec{m}) P(\vec{m})
\end{aligned}
$$


where here (and whenever it appears in the following) the sum over $\vec{m}$ is performed on $\vec{m} \in\left(Z_{N}^{+}\right)^{N}$ and

$$
P(\vec{m}):=\prod_{j=1}^{N}\left(1-q^{j-1}\right)^{m_{j}} .
$$

Let also

$$
e_{j}:=\sum_{a=1}^{M} c_{i, a} \delta_{j, d_{a}}
$$

with this notation and using (39) and (40) it is immediate to check that

$$
M+B_{M, C_{N, M}, p}=\tilde{B}_{p}\left(f_{M, C_{N, M}}\right)
$$

where, for any couple $\left(M, C_{N, M}\right), f_{M, C_{N, M}}$ : $\left(Z_{N}^{+}\right)^{N} \rightarrow(0,1 / N, \ldots N / N)$ is defined by

$$
f_{M, C_{N, M}}(\vec{m}):=N^{-1} \sum_{i=1}^{N} \prod_{j=1}^{N} \delta_{m_{j}, e_{j}}
$$

and

$$
\begin{aligned}
& \tilde{B}_{p}(f):= \\
& N \sum_{\vec{m}} f(\vec{m})\left[\sum_{i=1}^{N} \frac{m_{i}}{i}+q 0^{m_{1}} \exp \left(-\sum_{i=2}^{N} m_{i} a_{p}^{i}\right)\right]
\end{aligned}
$$

Therefore by using (42) and definition (26) we get

$$
\begin{gathered}
\min _{M, C_{N, M}}\left(M+B_{M, C_{N, M}, p}\right)=\min _{M, C_{N, M}} \tilde{B}_{p}\left(f_{M, C_{N, M}}\right) \\
\geq \inf _{f \in \mathcal{F}} \tilde{B}_{p}(f) \geq N \min _{\vec{m} \in\left(Z_{N}^{+}\right)^{N}} A_{p}(\vec{m})=N \bar{A}_{p}
\end{gathered}
$$

where $\mathcal{F}$ is the set of probability functions on $\left(Z_{N}^{+}\right)^{N}$, i.e. $f:\left(Z_{N}^{+}\right)^{N} \rightarrow \mathbb{R}^{+}$with $\sum_{\vec{m}} f(\vec{m})=$ 1. The second inequality immediately follows from the definition (26) and the fact that $f$ is a probability distribution.

The following remark will be used to construct an optimal algorithm in section 4

Remark 2: Define $g \in \mathcal{F}$ as

$$
g(\vec{m}):= \begin{cases}1 & \text { if } \vec{m}=\bar{m} \\ 0 & \text { otherwise }\end{cases}
$$

with $\bar{m} \in\left(\mathbb{Z}^{+}\right)^{N}$ such that

$$
\bar{m}_{i}:= \begin{cases}{[|\log p| / \log 2]} & \text { if } i=[\log 2 / p] \\ 0 & \text { otherwise }\end{cases}
$$

Then:

$$
\frac{\tilde{B}_{p}(g)}{N p|\log p|}=\frac{1}{(\log 2)^{2}}+o(p) .
$$

Furthermore $g$ coincides with $f_{M, C_{N, M}}$ (43) on all bipartite graphs with $M=N\left[p|\log p| /(\log 2)^{2}\right]=\bar{M}$ tests and connectivity matrix $C_{N, M}$ such that the number of tests per variable is fixed equal to $[|\log p| / \log 2]=\bar{L}$.

\subsection{Proof of Lemma 3 and Claim 1}

Proof of Lemma 3 . Let $\bar{m}$ be the vector over which $\bar{A}_{p}$ is reached. We consider separately the two complementary cases: (a) $\bar{m}_{1} \geq 1$ and (b) $\bar{m}_{1}=0$. In case (a), the minimum is obviously larger or equal to one. Therefore

$$
\bar{A}_{p} \geq \min \left(1, b_{p}\right)
$$

where

$$
b_{p}:=\min _{\substack{\vec{m} \in\left(Z_{N}\right)^{N} \\ m_{1}=0}} A_{p}(\vec{m})
$$

We now enlarge the minimization of $m_{i}$ to all real positive values $\mathbb{R}^{+}=[0, \infty)$, and introduce the two functions on $\left(\mathbb{R}^{+}\right)^{N-1}$ :

$$
\begin{aligned}
u(\vec{m}) & :=\sum_{i=2}^{N} m_{i} / i \\
v_{p}(\vec{m}) & :=\sum_{i=2}^{N} m_{i} a_{p}^{i}
\end{aligned}
$$

A simple bound on $b$ is expressed in terms of these functions:

$$
b_{p} \geq \min _{\vec{m} \in\left(\mathbb{R}^{+}\right)^{N-1}}\left(u(\vec{m})+(1-p) e^{-v_{p}(\vec{m})}\right) .
$$

This minimization is carried out in two steps. We first fix $v_{p}(\vec{m})=w \geq 0$, and look for the minimum of $u$ in the subspace $v_{p}(\vec{m})=w$. Let us denote by $u^{*}(w, p)$ this minimum value. Finding $u^{*}$ is a problem of linear optimization. So the minimum must be obtained on one of the vertices of the simplex of $\left(\mathbb{R}^{+}\right)^{N-1}$ defined by $v_{p}(\vec{m})=w$. These vertices are easily identified: There are $N-1$ of them, located at points $\vec{m}^{(2)}, \ldots, \vec{m}^{(N)}$, with $m_{j}^{(r)}=\delta_{j, r} w / a_{p}^{r}$. As $u\left(\vec{m}^{(r)}\right)=w /\left(r a_{p}^{r}\right)$, the minimum of $u$ is at $u^{*}(w, p)=w \min _{r \in\{2, \ldots, N\}} 1 /\left(r a_{p}^{r}\right)$. By enlarging 
the space of $r$ to all real values in $[2, \infty)$, we get:

$$
u^{*}(w, p) \geq w U(p) .
$$

Now we carry the optimization in (52) as:

$$
b_{p} \geq \min _{w \in[0, \infty)}\left[w U(p)+(1-p) e^{-w}\right]
$$

which establishes lemma 3 .

$$
\text { Proof of Claim 1) Let } z:=(1-p)^{r-1} \text { and }
$$

$$
g_{p}(z):=\frac{(1-z) \log (1-z)}{z \log [z(1-p)]} .
$$

For $r \geq 2$ and $0<p<1$, it is immediate to verify that $0<z \leq(1-p)$ and that any stationarity point for $U(p)$ must satisfy $g_{p}(z)=1$. By studying the function $g_{p}(z)$ it is then possible to prove that there are two values $z \in(0,1)$ which satisfy the latter condition. Furthermore, when $p \rightarrow 0$ only one of these two values belongs to $(0,1-p]$ and it corresponds to $z=1 / 2-\epsilon(p)$ with $\epsilon(p)=\Theta(p)$. The desired result for $U(p)$ immediately follows.

4 UPPER BOUNDS ON $\bar{T}(N, p)$ FOR $\beta \in$ $[0,1 / 2)$ VIA REGULAR-REGULAR GRAPHS

In this section we prove Theorem 3 , On the one hand this result allows to complete the proof of Theorem 11, namely to identify the sharp asymptotic value of $\bar{T}(N, p) /(N p|\log p|)$ in the limit $N \rightarrow \infty, p=N^{-\beta}$ for $\beta \in[0,1 / 2)$. Precisely,

Proof of Theorem 1; The proof follows immediately from Theorem 6 and Theorem 3 .

On the other hand Theorem 3 provides a constructive procedure for a class of algorithms (i.e. a choice of $M$ and a class of matrices $\left.\left\{C_{N, M}\right\}\right)$ which are asymptotically optimal.

In order to construct these algorithms we will keep in mind the following observations. First, as already remarked in the previous section, the number of tests due to the undetermined ones is negligible for all the choices of $p$ discussed in Theorem 3. Therefore we focus on algorithms that minimize the number of tests in the first stage, $M$, plus the number of undetermined zeros, $\left|\mathcal{U}_{0}\right|$. The second observation is that inequality (33) comes from (31) and the latter becomes an equality provided $M, C_{N, M}$ are such that the corresponding graph has girth at least 6 . The third observation is the one contained in remark 2 which states that the minimum for the right hand side of (33) is attained on any graph with $\bar{M}$ tests and $\bar{L}$ tests per variable, where $\bar{M}$ and $\bar{L}$ have been defined in (10) and (11) (we recall that both $\bar{M} / N$ and $\bar{L}$ depend only on $p$ ). Therefore if it is possible to find at least one graph with girth at least 6 among those with $\bar{M}$ tests and $\bar{L}$ tests per variable, the mean number of tests on this graph will match the lower bound in Theorem 6 in the limit $p \rightarrow 0$. In the following we will use the above ideas and the results on regular-regular graphs with a fixed minimal girth which have been obtained in [17].

Proof of Theorem 3. Consider a connectivity matrix $C_{N, \bar{M}}^{\bar{L}}$ with fixed variable degree $\bar{L}$, fixed test degree $\bar{K}=\bar{L} N / \bar{M}$ and girth at least 6 (see condition (56)). The proof of the existence of such a graph and an explicit procedure for its construction have been provided by $\mathrm{Lu}$ and Moura in their study of large girth LDPC codes [17]. Their procedure requires that the condition

$$
\bar{M} \geq \frac{(\bar{L}-1)(N \bar{L} / \bar{M})}{\overline{L K}-\bar{L}-\bar{K}}
$$

be satisfied. (This condition corresponds to condition (14) in appendix A of [17] for the choice $g=6$ ). In the limit $N \rightarrow \infty$ with $p=1 / N^{\beta}$ with $\beta<1 / 2$, the validity of (56) can be readily checked, using definitions (10) and (11).

From equation (2) and (37), the number of tests on any such graph satisfies the inequality

$$
\lim _{N \rightarrow \infty} T_{\bar{M}, C_{N, \bar{M}}^{\bar{L}}, p} \leq \bar{M}+N p+N(1-p) R_{p}
$$

where

$$
R_{p}=\sum_{X} \mu_{p}(X) \prod_{a=1}^{\bar{M}} W_{i, a}=\left(1-(1-p)^{\bar{K}-1}\right)^{\bar{L}} .
$$

The last equality is obtained by using definition (38) for $W_{i, a}$ (the mean over the Bernoulli distribution is easily performed thanks to the girth condition). Theorem 3 immediately follows from (57). 
The results (57) and (58) hold for any choice of $\mathrm{p}$. However, it is important to notice that the existence of at least one such connectivity matrix with girth at least 6 is guaranteed only for $\beta \in[0,1 / 2)$. In particular for $p=N^{-\beta}$ with $1<\beta<2$ there cannot exist any such matrix: otherwise (57) and (58) would imply $\bar{T}(N, p) \leq \beta /(\log 2)^{2} N^{1-\beta} \log N$ which goes to zero as $N \rightarrow \infty$ (and is in contradiction with the lower bounds in formula (57) of [14]).

Putting together (57), (58) and (36) it is also immediate to verify that the higher order corrections to the optimal value $\bar{T}(N, p)$ are of order $p N$. More precisely, if we let

$$
H(N, p):=\frac{\bar{T}(N, p)-N p|\log p|(\log 2)^{-2}}{N p}
$$

the following holds.

Remark 3: For $\beta \in[0,1 / 2)$ in the limit $N \rightarrow$ $\infty$ the following holds

$$
\frac{1-2|\log \log 2|}{(\log 2)^{2}} \leq H(N, p) \leq 2
$$

\section{Regular-Poisson graphs are (ALSO) OPTIMAL FOR $\beta \in[0,1 / 2)$}

In this section we will prove Theorem 4 which shows that asymptotically optimal pool designs are obtained with regular-Poisson distributions for proper choices of the graph parameters. This is particularly relevant since the construction of regular-Poisson graphs is much simpler than the construction of [17] for regular-regular graphs with girth at least 6 .

Consider the regular-Poisson distribution on bipartite graphs defined in section 2 with $N$ variable nodes, $\bar{M}$ test nodes and $\bar{L}$ tests per variable, $P_{N, \bar{M}}^{\mathcal{R}-\mathcal{P}}$. Fix a variable, $i$, and let $\mathcal{E}_{i}^{n}$ be the characteristic function of the event (defined over the space of all bipartite graphs with $\bar{M}$ nodes) that there are more than $n$ loops of length 4 which contain $i$, i.e. there are more than $n$ triples $(j, a, b)$ with $j$ a variable different from $i(j \neq i, j \in(1, \ldots, N))$ and $a, b$ two distinct tests $(a \neq b, a, b \in(1, \ldots \bar{M}))$ such that $i$ and $j$ belong to both tests. Precisely, we define $\mathcal{E}_{i}^{n}: C_{N, \bar{M}} \in\{0,1\}^{N \times \bar{M}} \rightarrow \mathbb{R}$ as $\mathcal{E}_{i}^{n}\left(C_{N, \bar{M}}\right):=1$ if

$$
\sum_{j=1,(j \neq i)}^{N} \sum_{1 \leq a<b \leq M} c_{i, a} c_{i, b} c_{j, b} c_{j, a}>n
$$

and $\mathcal{E}_{i}^{n}\left(C_{N, \bar{M}}\right):=0$ otherwise.

In order to prove Theorem 4 we will need the following Lemmas which give an upper bound on the probability that there are more than $n$ loops of length 4 through $i$ (Lemma 4) and an upper bound on the probability that $i$ is an undetermined zero and does not belong to more than $n$ loops of length 4 (Lemma [5).

Lemma 4:

$$
\begin{aligned}
& \sum_{C_{N, \bar{M}}} P_{N, \bar{M}, \bar{L}}^{\mathcal{R}-\mathcal{P}}\left(C_{N, \bar{M}}\right) \mathcal{E}_{i}^{n}\left(C_{N, \bar{M}}\right) \leq \\
& \leq \frac{N \bar{L}^{6}}{\bar{M}^{3}}+\left(\frac{N \bar{L}^{4}}{\bar{M}^{2}}\right)^{n+1} .
\end{aligned}
$$

Lemma 5: Let $\bar{k}$ be the average degree of the checks, $\bar{k}:=N \bar{L} / \bar{M}=\log 2 / p$, and

$$
C_{p}:=\sum_{C_{N, \bar{M}}} P_{N, \bar{M}, \bar{L}}^{\mathcal{R}-\mathcal{P}}\left(C_{N, \bar{M}}\right)\left(1-\mathcal{E}_{i}^{n}\right) \sum_{X} \mu_{p}(X) \prod_{a=1}^{\bar{M}} W_{i, a}
$$

(we drop for semplicity of notation the dependence of $W_{i, a}$ on $X, C_{N, \bar{M}}$ and the dependence of $\mathcal{E}_{i}^{n}$ on $\left.C_{N, \bar{M}}\right)$. Define also $\gamma:=p^{\alpha}$.

For any $n$ and $\alpha$ with $0<n<\bar{L} / 2$ and $0<$ $\alpha<1 / 2$, the following holds

$$
\begin{aligned}
& C_{p} \leq\left[1-(1-p)^{\bar{k}(1+\gamma)}\right]^{\bar{L}-2 n}+ \\
& +\bar{L} \exp \left[-\frac{\gamma^{2} \log 2}{2 p}\right]+o\left(\bar{L} \exp \left[-\frac{\gamma^{2} \log 2}{2 p}\right]\right) .
\end{aligned}
$$

\section{Proof of Theorem 4:}

For any $n$ and $\alpha$ with $0<n<\bar{L} / 2$ and $0<$ $\alpha<1 / 2$, the mean number of tests verifies

$$
\begin{aligned}
& \sum_{C_{N, \bar{M}}} P_{N, \bar{M}, \bar{L}}^{\mathcal{R}-\mathcal{P}}\left(C_{N, \bar{M}}\right) T_{\bar{M}, C_{N, \bar{M}}, p} \leq \bar{M}+N p+ \\
& \frac{|\log p|^{3}}{N p^{3}}+N\left(\frac{|\log p|^{2}}{N p^{2}}\right)^{n+1}+N\left[1-(1-p)^{\bar{k}(1+\gamma)}\right]^{\bar{L}-2 n} \\
& +N \bar{L} \exp \left[-\frac{\gamma \log 2}{2 p}\right]+N_{O}\left(\bar{L} \exp \left[-\frac{\gamma \log 2}{2 p}\right]\right)
\end{aligned}
$$

where $\gamma:=p^{2 \alpha}$.

In order to derive (65) we have: (i) used definition (2); (ii) bounded the mean number of undetermined ones with the mean number 
of ones; (iii) decomposed the mean number of undetermined zeros into those that are (are not) on a variable node $i$ which contain more than $n$ loops of length 4; (iv) upper bounded the last two terms via the results of Lemma 4 and Lemma 5, If we now make the choice $n:=\bar{L}^{-1 / 2}$ the result of the Theorem immediately follows by noticing that:

$$
\begin{aligned}
& \lim _{N \rightarrow \infty \mid \beta} \frac{N \bar{L} \exp \left(-\gamma^{2} p^{-1} \log 2 / 2\right)}{N p|\log p|}=0 \\
& \lim _{N \rightarrow \infty \mid \beta} \frac{|\log p|^{3}}{N p^{3} N p|\log p|}=0 \\
& \lim _{N \rightarrow \infty \mid \beta} \frac{N}{N p \log p}\left(\frac{|\log p|^{2}}{N p^{2}}\right)^{n+1}=0 .
\end{aligned}
$$

Proof of Lemma 44 Given a connectivity matrix, $C_{N, \bar{M}}$, we identify among the loops of length 4 two distinct classes: loops of type $S$ and of type $D$. Loops of type $S$ are those disconnected from any other loop, namely they correspond to the choices of two variables $i, j$ and two tests $a, b$ such that both $i$ and $j$ belongs to $a$ and $b$ and there does not exist another test containing both $i$ and $j$. Loops of type $D$ are all loops of length 4 which are not of type $S$. For a given variable $i$, let $\mathcal{D}_{i}$ be the characteristic function of the event that $i$ belongs to at least one loop of type $D$. Precisely, we define $\mathcal{D}_{i}: C_{N, \bar{M}} \in\{0,1\}^{N \times \bar{M}} \rightarrow \mathbb{R}$ as $\mathcal{D}_{i}\left(C_{N, \bar{M}}\right):=1$ if

$$
\sum_{j=1,(j \neq i)}^{N} \sum_{1 \leq a<b<c \leq M} c_{i, a} c_{i, b} c_{i, c} c_{j, b} c_{j, a} c_{j, c}>0
$$

and $\mathcal{D}_{i}\left(C_{N, \bar{M}}\right):=0$ otherwise.

The following inequalities hold

$$
\begin{aligned}
& \sum_{C_{N, \bar{M}}} P_{N, \bar{M}, \bar{L}}^{\mathcal{R}-\mathcal{P}}\left(C_{N, \bar{M}}\right) \mathcal{D}_{i} \leq \frac{N \bar{L}^{6}}{\bar{M}^{3}} \\
& \sum_{C_{N, \bar{M}}} P_{N, \bar{M}, \bar{L}}^{\mathcal{R}-\mathcal{P}}\left(C_{N, \bar{M}}\right) \mathcal{E}_{i}^{n}\left(1-\mathcal{D}_{i}\right) \leq\left(\frac{\bar{L}^{4} N}{\bar{M}^{2}}\right)^{n+1}
\end{aligned}
$$

(we drop the dependence of $\mathcal{D}_{i}$ and $\mathcal{E}_{i}{ }^{n}$ on $C_{N . \bar{M}}$.) The result follows immediately from (70) and (71) and the fact that, for any $i$ and $C_{N, \bar{M}}, \mathcal{E}_{i}{ }^{n}\left(C_{N, \bar{M}}\right)<1$.

Proof of Lemma 5. For a given connectivity matrix $C_{N, \bar{M}}$, let $i$ be a site with less than $n$ loops of length 4 . Let us call $A_{i}\left(C_{N, \bar{M}}\right)$ the set of tests which contain $i, B_{i}\left(C_{N, \bar{M}}\right)$ the set of tests which belong to a loop of length 4 passing through $i$, and $\bar{B}_{i}\left(C_{N, \bar{M}}\right)=A_{i}\left(C_{N, \bar{M}}\right) \backslash B_{i}\left(C_{N, \bar{M}}\right)$. Clearly $\left|B_{i}\left(C_{N, \bar{M}}\right)\right| \leq 2 n$. The following holds

$$
\begin{aligned}
& \prod_{a=1}^{\bar{M}} W_{i, a} \leq \prod_{a \in \bar{B}_{i}\left(C_{N, \bar{M}}\right)} W_{i, a} \\
& =\prod_{a \in \bar{B}_{i}\left(C_{N, \bar{M}}\right)}\left(1-\prod_{\substack{j=1, \ldots, N \\
j \neq i}}\left(1-x_{j}\right)^{c_{j a}}\right) .
\end{aligned}
$$

We can now plug (72) into (63) and get

$$
\begin{aligned}
& C_{p} \leq \sum_{C_{N, \bar{M}}} P_{N, \bar{M}, \bar{L}}^{\mathcal{R}-\mathcal{P}}\left(C_{N, \bar{M}}\right) \\
& \mathbb{I}\left(\left|\bar{B}_{i}\left(C_{N, \bar{M}}\right)\right| \geq \bar{L}-2 n\right) \prod_{a \in \bar{B}_{i}\left(C_{N, \bar{M}}\right)}\left[1-(1-p)^{d_{a}-1}\right]
\end{aligned}
$$

where in order to perform the mean over $\mu(X)$ we used the fact that the neighborhoods of any two tests $a, b$ belonging to $\bar{B}_{i}\left(C_{N, \bar{M}}\right)$ intersect only in $i$ (for any $j \neq i$ one has $\left.c_{j a} c_{j b}=0\right)$ and we recall from definition (27) that $d_{a}$ is the degree of test $a$. Let $k_{\max }$ be the maximum degree of the first $\bar{L}$ tests, namely $k_{\text {max }}:=\max _{a \in(1, \ldots, \bar{L})} \sum_{j} c_{j, a}$. Using (75) and the invariance of the regular-Poisson distribution under test permutations, we get

$$
\begin{aligned}
C_{p} & \leq \sum_{C_{N}, \bar{M}} P_{N, \bar{M}, \bar{L}}^{\mathcal{R}-\mathcal{P}}\left(C_{N, \bar{M}}\right) \sum_{k=0}^{N} \delta_{k, k_{\max }}\left(1-(1-p)^{k}\right)^{\bar{L}-2 n} \\
& \leq\left(1-(1-p)^{\bar{k}(1+\gamma)}\right)^{\bar{L}-2 n}+\bar{L} G_{p}
\end{aligned}
$$

where

$G_{p}:=$

$\sum_{k=\bar{k}(1+\gamma)}^{N}\left(\begin{array}{l}N \\ k\end{array}\right)\left(\frac{\bar{k}}{N}\right)^{k}\left(1-\frac{\bar{k}}{N}\right)^{N-k}\left(1-(1-p)^{k}\right)^{\bar{L}-2 n}$.

It is now easy to verify that in the limit $p \rightarrow 0$ :

$G_{p} \leq \exp \left[-\gamma^{2} p^{-1} \log 2 / 2\right]+o\left(\exp \left[-\gamma^{2} p^{-1} \log 2 / 2\right]\right)$

and by plugging (77) into (75) the proof is completed. 


\section{UPPER BOUNDS ON $\bar{T}(N, p)$ FOR $\beta \in$ $[1 / 2,1)$ VIA POISSON-POISSON GRAPHS}

In this section we prove Theorem 5, This allows to complete the proof of Theorem 2 which establishes upper and lower bounds on $\bar{T} /(N p|\log p|)$ when $p=1 / N^{\beta}$ and $1 / 2 \leq \beta<1$.

Proof of Theorem 2. The proof follows immediately from Theorem 6 and Theorem 5.

Furthermore Theorem 5 allows to identify a class of algorithms over which the upper bound is attained.

Proof of Theorem 5. Consider the class of Poisson-Poisson distributions on bipartite graphs defined in (19) with $N$ variable nodes, $M$ test nodes, a mean number of tests per variable equal to $L$ and a mean number of variables per test equal to $K=N L / M, P_{N, M, L}^{\mathcal{P}-\mathcal{P}}$. From (37), performing first the average with respect to the Poisson-Poisson distribution in which the $c_{i a}$ variables are iid, the mean number of undetected zeros can be written as:

$$
\begin{aligned}
& \sum_{C_{N, M}} P_{N, M, L}^{\mathcal{P}-\mathcal{P}}\left(C_{N, M}\right) \sum_{X} \mu_{p}(x)\left|\mathcal{U}_{0}\right|= \\
& N \sum_{X} \mu_{p}(x)\left(1-x_{1}\right)\left[1-\frac{K}{N} \prod_{j=2}^{N}\left(1-x_{j} \frac{K}{N}\right)\right]^{M} .
\end{aligned}
$$

Denoting by $r$ the number of indices $j$ such that $x_{j}=1$, this gives:

$$
\begin{aligned}
& \sum_{C_{N, M}} P_{N, M, L}^{\mathcal{P}-\mathcal{P}}\left(C_{N, M}\right) \sum_{X} \mu_{p}(x)\left|\mathcal{U}_{0}\right|= \\
& N \sum_{r=0}^{N-1}\left(\begin{array}{c}
N-1 \\
r
\end{array}\right) p^{r} q^{N-r}\left(1-\frac{K}{N}\left(1-\frac{K}{N}\right)^{r}\right)^{M}
\end{aligned}
$$

where we recall that $q:=(1-p)$.

Let $\gamma:=p /|\log p|$, then

$$
\begin{aligned}
& \sum_{r=N(p+\gamma)}^{N-1}\left(\begin{array}{c}
N-1 \\
r
\end{array}\right) p^{r}(1-p)^{N-r} \\
& <\exp \left[-N \gamma^{2} p^{-1} / 2\right]+o\left(\exp \left[-N \gamma^{2} p^{-1} / 2\right]\right) .
\end{aligned}
$$

By using definition (2) and the above equations
(78), (79) and (80) we get

$$
\begin{aligned}
& \lim _{N \rightarrow \infty \mid \beta} \sum_{C_{N, M}} P_{N, M, L}^{\mathcal{P}-\mathcal{P}}\left(C_{N, M}\right) \frac{T_{M, C_{N, M}, p}}{N p|\log p|} \leq \\
& \lim _{N \rightarrow \infty \mid \beta}(p|\log p|)^{-1}\left[\frac{M}{N}+\left(1-\frac{K}{N}\left(1-\frac{K}{N}\right)^{N p+N \gamma}\right)^{M}\right. \\
& \left.+N^{-1} \exp \left(-3 N p|\log p|^{-2} / 2\right)+p\right]= \\
& \lim _{N \rightarrow \infty \mid \beta}\left[\frac{M}{N p|\log p|}+\frac{\left(1-\frac{K}{N}\left(1-\frac{K}{N}\right)^{N p+N \gamma}\right)^{M}}{p|\log p|}\right] .
\end{aligned}
$$

By minimizing the last expression on $M$ and $K$ we find that the optimal value is taken on $M=e p N|\log p|+o(N p|\log p|)=\widetilde{M}+o(\widetilde{M})$ and $K=1 / p+o(1 / p)=N \widetilde{L} / \widetilde{M}+o(N \widetilde{L} / \widetilde{M})$, where $\widetilde{L}$ and $\widetilde{M}$ have been defined in (20) and (21). Furthermore

$$
\lim _{N \rightarrow \infty \mid \beta} \sum_{C_{N, M}} P_{N, \widetilde{M}, \widetilde{L}}^{\mathcal{P}-\mathcal{P}}\left(C_{N, M}\right) \frac{\bar{T}(N, p)}{N p|\log p|} \leq e
$$

is easily verified, thus completing the proof of Theorem 2 .

Remark 1 can be proven along the same lines.

\section{ACKNOWLEDGMENT}

We thank Gregory Sorkin and Irina Rish for interesting discussions which stimulated our interest in the group testing problem

\section{REFERENCES}

[1] R.Dorfman, "The detection of defective members of large poulations," Ann.Math.Statist., vol. 14, pp. 436-440, 1943.

[2] S.A.Zenios and L.M.Wein, "Pooled testing for hiv prevalence estimation: Exploiting the dilution effect," Stat.Med., vol. 17, pp. 1447-1467, 1998.

[3] B. E.Barillot and D.Cohen, "Theoretical analysis of library screening using and $n$-dimensional pooling strategy," Nuc.Acids Res, vol. 19, pp. 6241-6247, 1991.

[4] E. D. C. N. R. W.J.Bruno, D.J.Baldings and D.C.Torney, "Design of efficient pooling experiments," Genomics, vol. 26, pp. 21-30, 1995.

[5] D.Margaritis and S.Skiena, "Reconstructing strings from substrings in rounds," Proc.Found.Comput.Sci., pp. 613620, 1995.

[6] P.A.Pevzner and R.Lipshutz, "Toward dna sequencing chips," in Proc.19th Int.conf.Math.Found.Comput.Sci., Lecture notes on computer sciences, 1994, pp. 143-158.

[7] M.Sobel and P.A.Groll, "Group testing to eliminame efficiently all defectives in a binomial sample," Bell System tech. J., vol. 28, pp. 1179-1252, 1959. 
[8] W.H.Kautz and R.C.Singleton, "Nonrandom binary superimoposed codes," IEEE Trans.on Information Th., vol. 10, pp. 363-377, 1964.

[9] E.H.Hong and R.E.Ladner, "Group testing for image compression," IEEE Trans.on Image Proc., vol. 11, pp. 901-911, 2002.

[10] Y.W.Hong and A.Scaglione, “On multiple access for distributed dependent sensors: A content-based froup testing approach," Proc. IEEE Inf.Theory Workshop, pp. 298-303, Oct 24-29 2004.

[11] D.Z.Du and F.K.Hwang, Combinatorial Group Testing and its Applications. Singapore: World Scientific, 2000.

[12] E. D.J.Balding, W.J.Bruno and D.C.Torney, A comparative survey of nonadaptive pooling designs. New York: T.S Speed and M.Waterman Eds., Springer Verlag, 1996, pp.133-154.

[13] E.Knill, "Lower bounds for identifying subset members with subset queries," in Proc.6th Ann.ACM-SIAM Symp.discr.Algorithms, San Francisco, Jan. 1995, pp. 369377.

[14] T.Berger and V.I.Levenshtein, "Asymptotic efficiency of two-stage disjunctive testing," IEEE Trans. on Inf.Th., vol. 48, pp. 1741-1749, 2002.

[15] L. A.De Bonis and U.Vaccaro, "Optimal two-stage algorithms for group testing problems," SIAM Journal on Computing, vol. 34, pp. 1253-1270, 2005.

[16] T.Berger and V.I.Levenshtein, "A universal bound for a covering in regular posets and its application to pool testing," Discrete Applied Mathematics, vol. 128, pp. 11-26, 2003.

[17] J.Lu and J.M.F.Moura, "Structured ldpc codes for highdensity recording: large girth and low error flow," IEEE Trans. on Magnetics, vol. 42, pp. 208-213, 2006.

[18] P. C.M.Fortuin and J.Ginibre, "Correlation inequalities on some partially ordered sets," Comm.Math.Phys., vol. 22, pp. 89-103, 1971. 